\title{
Lewis Acid Combined with Microwave Converts Hemicellulose and Cellulose in Cane Pith into 5-Hydroxymethyl furfural and Glucose
}

\author{
Kun $\mathrm{du}^{*}$, Yufeng Zeng and Ronghuan Qin \\ College of Chemistry and Food Science, Yulin Normal University, Yulin, Guangxi 537000, China
}

\begin{abstract}
The paper using sugarcane pith as a raw material, the feasibility of aluminum hydroxide Lewis acid combined with microwave conditions to be used as a resource was investigated.Under the conditions of the combination of stellate acid and microwave, the 5-hydroxymethyl furfural (5-HMF) in $0.9 \%$ of the liquid phase product can be detected, and its low yield of $0.9 \%$ is worthy of further improvement. Economic analysis shows that the added value of 5-HMF produced by Lewis acid combined with microwave is as high as about 30,000 yuan per ton, and the prospect of resource utilization is huge. Use oxalic acid to hydrolyze the remaining cellulose and hemicellulose after 5-HMF is prepared from sugarcane pith to glucose, which is used in food and related products. It has very important application value in the industry. In this paper, the hemicellulose in cane pith is converted to 5-HMF under microwave reaction conditions, and then the remaining residue of the reaction is hydrolyzed with oxalic acid to produce glucose, which provides theoretical basis and technical support for further research on the high-value utilization of sugar cane pith in the future.
\end{abstract}

Key words: Aluminum hydroxide Lewis acid; 5-hydroxymethyl furfural; Oxalic acid; Glucose.

\section{Introduction}

Bagasse is a by-product of the sugarcane sugar industry, a rich and cheap renewable resource. Bagasse has now become one of the main raw materials for papermaking, especially in recent years Guangxi has vigorously developed bagasse papermaking, and the output of bagasse pulp has been increasing year by year. Major sugar mills have established their own bagasse pulp and paper mills to implement the strategy of combining sugar and paper to improve the economic benefits of enterprises. Research and analysis have shown that bagasse contains $32 \sim 48 \%$ cellulose, $19 \sim 24 \%$ hemicellulose, and $23 \sim$ $32 \%$. Lignin, and about $4 \%$ ash.

When using bagasse to make paper, it is necessary to separate the sugarcane pith that affects the quality of the paper. Guangxi alone produces 2.5 to 3 million tons of sugarcane pith a year. The composition of sugarcane pith is similar to that of bagasse. The main components are cellulose $32 \%-40 \%$, hemicellulose $24 \%-29 \%$, and lignin $27 \%-32 \%$. It is one of the few plant fibers with extremely high hemicellulose content. The main component of hemicellulose is polypentose, which is easily hydrolyzed into monosaccharides such as xylose, L-arabinose and glucose by dilute acid. At present, the processing of sugarcane pith in Guangxi Sugar Factory is basically burned as fuel or discarded directly. It not only causes environmental pollution but also makes the added value of sugarcane pith very low. and the added value is very low. With the rapid growth of fossil energy consumption and the depletion of its reserves, the development and application of renewable clean energy has attracted much attention. In the report published in 2004, 12 platform molecules of biomass sugars were put forward for the first time, and the "concept of biomass refining" was emphasized. Among them, the awakening of the sleeping giant 5-hydroxymethylfurfural (5-HMF) brings furan compounds and polymers back to the forefront of basic research and industry. After Leshko et al. [1] published an article on the efficient production of 5hydroxymethylfurfural in a two-phase organic reaction system using fructose as a substrate in Science, 5-HMF formally entered people's attention. Using 5-HMF as a raw material can produce a series of downstream products. 5 -HMF is prepared by removing three molecules of water from monosaccharides through catalytic reactions [2]. Disaccharides and polysaccharides such as sucrose, cellobiose, cellulose, etc. can also be used as raw materials to prepare 5-HMF, but firstly, it must undergo a hydrolysis process to depolymerize it into monosaccharides. Although the method seems simple, 5HMF is easy to rehydrate levulinic acid and formic acid during the reaction, and cross-polymerize into soluble polymers and insoluble humic substances, which

\footnotetext{
* Corresponding author: huatangchun769@126.com
} 
complicates the synthesis process of 5-HMF [3]. At present, researchers have proposed a variety of reaction mechanisms for the dehydration of hexose to produce 5HMF based on extensive research, which can be roughly divided into pathway 1 and pathway 2, as shown in Figure 1 .

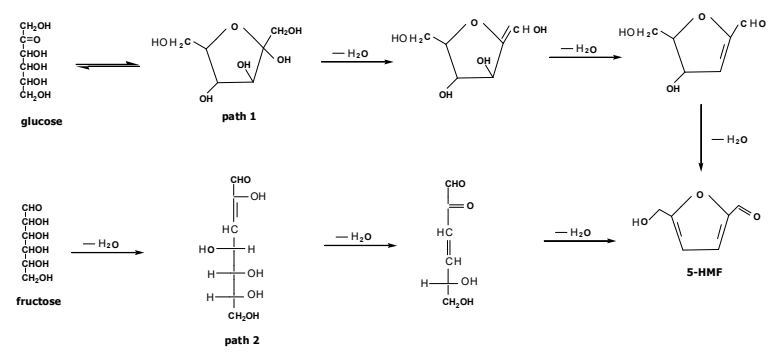

Figure 1. The mechanism of dehydration of glucose and fructose to produce 5-HMF.

It can be seen from Figure 1 that the biggest difference between the two routes lies in whether or not intermediates of cyclic compounds are generated during the conversion of monosaccharides to 5-HMF. In Path 1, glucose is first converted to fructose through isomerization, and then fructose can generate specific intermediates under certain conditions, and further dehydration to generate 5-HMF, while path $\mathrm{B}$ directly obtains 5-HMF by removing three molecules of water. At present, more research results indicate that the main mechanism of hexose dehydration to produce 5-HMF is path 1. Amarasekara et al. [4] used ${ }^{1} \mathrm{H}-\mathrm{NMR}$ and ${ }^{13} \mathrm{C}$ NMR to dehydrate D-fructose in DMSO solvent to produce 5-HMF, and detected the existence of a key intermediate in Path 1.

From the above discussion, monosaccharides such as fructose and glucose are the direct precursors for the preparation of 5-HMF. However, the content of this series of substances in plant solid waste is often very low. Plant solid waste is more challenging than monosaccharide carbohydrates such as fructose and glucose to produce 5HMF as a raw material due to its high polymerization and difficult decomposition characteristics. Therefore, from the perspective of raw material selection, plant solid wastes with high content of monosaccharides, cellulose, and hemicellulose have higher 5-HMF conversion potential. Without adding a catalyst, a raw material with a high proportion of hemicellulose is easier to prepare 5HMF[5] [6] .

The research using Lewis acid (L acid) as a catalyst is the most outstanding research result of Zhao et al. [7]. In 2007, Zhao et al. published their research results on Science. They found that Lewis acid in room temperature ionic liquid 1-ethyl-3-methylimidazole chloride can effectively catalyze the conversion of glucose to 5-HMF, and the reaction is complete. The yield of the latter 5-HMF is as high as $70 \%$.

As mentioned earlier, sugarcane pith is one of the few plant fiber materials with extremely high hemicellulose content. Sugarcane pith is a waste from the paper industry. Use this renewable resource reasonably, such as using it to prepare 5-HMF. The remaining residue contains a large amount of cellulose and can also be hydrolyzed to prepare glucose. It is of great practical significance and application value to increase the added value of sugarcane, turn waste into treasure, and reduce pollution, and it is urgent to discuss in depth. As described herein, the method of preparing 5-HMF from sugarcane pith using cheap Lewis acid aluminum hydroxide as a catalyst, and then continuing to use the residue, and then preparing glucose from it has not been reported in the relevant literature.

\section{Experiment}

\subsection{Use Jupiter-B Microwave Digester for Microwave Reaction}

The maximum microwave power and heating rate of Jupiter-B microwave digester are $800 \mathrm{~W}$ and $60^{\circ} \mathrm{C} / \mathrm{min}$, respectively. In the experiment, $1.000 \mathrm{~g}$ of sugarcane pith sample pretreated by grinding and sieving and $10 \mathrm{ml}$ of dilute aluminum hydroxide solution were added to each microwave tube, and then placed in the microwave instrument to carry out the conversion reaction of Lewis acid from glucose to fructose And the subsequent dehydration reaction of fructose.

After the reaction, the microwave tube was taken out of the microwave instrument and immediately placed in an ice-water bath to cool to terminate the reaction. After cooling, a Buchner funnel with quantitative filter paper is used to separate the microwave product from solid-liquid. The solid-phase product obtained from the separation is dried in an oven at $100^{\circ} \mathrm{C}$ to remove moisture, and sealed and stored for testing; liquid-phase products are temporarily stored for testing. The entire experimental process is shown in Figure 2: 


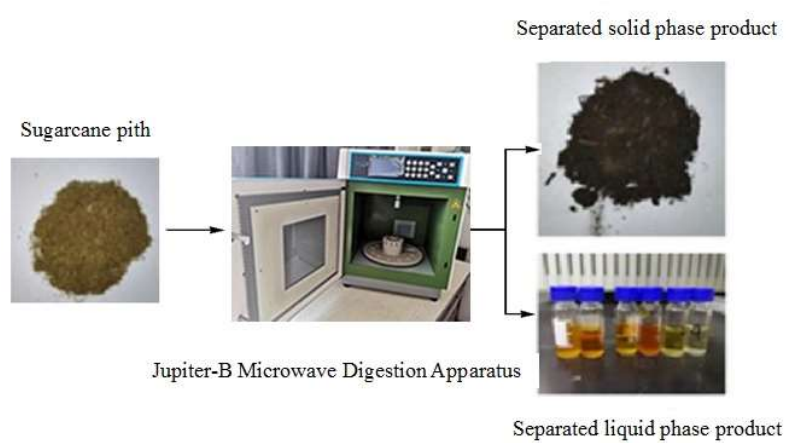

Figure 2. Sugarcane pith microwave-catalyzed conversion of glucose to fructose

The yield of 5-HMF: $\mathrm{Y}=100 \times \mathrm{CV} / \mathrm{M}$. Y is the yield of 5-HMF (\%), C is the HMF concentration $(\mathrm{mg} / \mathrm{L})$ detected by HPLC, $\mathrm{V}$ is the volume of the liquid product after constant volume (L), and $\mathrm{M}$ is the dry base of sugarcane pith (mg)

\subsection{Use Oxalic Acid to Hydrolyze the Residue for Producing Glucose}

Wash the sugarcane pulp residue, dry it, measure an appropriate amount of residue, add $75 \%$ oxalic acid in a double amount, and infiltrate it for 30 minutes. Then add about $1000 \mathrm{ml}$ of water, place it in a water bath to heat and boil for 1 hour, stirring occasionally. After heating, the product is suction filtered, and the filter residue is discarded. The filtrate is neutralized with calcium carbonate, the generated gypsum is filtered out, the filtrate (ie the sugar solution) is evaporated and concentrated, and then decolorized with activated carbon, and then the sugar solution is concentrated by vacuum distillation. Finally, the sugar solution is cleaned for three days, and the glucose is large. After crystallizing, drying, crushing, and weighing, the glucose is actually obtained, and the final glucose output is the ratio of the amount of glucose weighed after crushing to the residual dregs.

\section{Results and discussion}

In this study, a chromatographic scan was performed on the liquid product (Figure 3) which obtained from EXPERIMENT 1, and the valuable chemical 5-HMF was found through the comparison of standard materials. After further quantitative analysis, the concentration was $0.9 \%$.

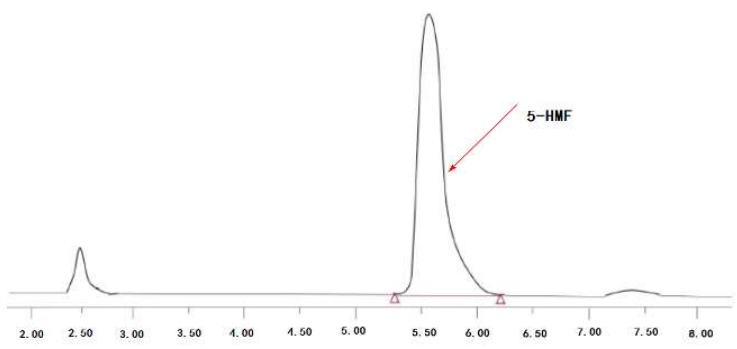

Figure 3. High performance liquid chromatogram of 5-HMF

5-HMF is composed of furan ring, hydroxymethyl group and aldehyde group. It is listed as one of the world's 12 most valuable chemicals. It is an important fine chemical raw material with a pure product value of 10,000 yuan $/ \mathrm{kg}$ (October 2019 data). The microwave hydrothermal liquid phase products of sugarcane pith are worthy of attention. The main properties of the prepared glucose powder which obtained from EXPERIMENT 2 was detected. Its color: white; dissolution: soluble in a small amount of water, clear and transparent; moisture: $9.10 \%$; glucose: 86.7\%; ash: 2.01; dextrin and other aldehydes $2.19 \%$. The ash is mainly gypsum, which has a slightly higher content, but gypsum can be completely removed by ion exchangers after the gypsum is filtered out during the concentration process.

\section{Conclusion}

Although the experiment obtained high purity 5-HMF, the high-value liquid phase product 5-HMF obtained in this experiment has a low yield. Related research has found that the formation process of 5-HMF is affected by many factors. It can be seen that 5-HMF, a microwave hydrothermal liquid phase product of sugarcane pith, has huge room for improvement.

The quality of sugarcane pulp residue is very good. It is saccharified with $75 \%$ oxalic acid to easily produce white glucose powder. The glucose yield is $53.3 \%$ to the residue. It is expected that improved operating conditions can also increase the yield.

\section{Acknowledgements}

The authors gratefully appreciate the financial support of the High-level Talent Research Startup Fund of Yulin Normal University through contract No. G2018024. 


\section{References}

1. Y. R. Leshkov, I. N. Chheda and J.A. Dumesic, Science 312(30): 1933-1937 (2006).

2. X. L. Tong, Y. Ma and Y. D. Li, Appl. Catal. A: Gen. 385 (1/ 2), 1-13 (2010).

3. A. Mukherjee, M. J. Dumont and V. Raghavan, BIOMASS BIOENERG. 72, 143-183 (2015).

4. R. Amar, A. Aseka, L. Williamsl, C . Ebede, Carbohydr. Res. 343(18): 3021-3024(2008).

5. B. K. Ozsel, D. Ozturk and B. Nis, Bioresour. Technol. 289: 121627-121633(2019).

6. Z. Chi, X. Zhao and T. Daugaard, BIOMASS BIOENERG. 120, 107-116 (2019).

7. H. Zhao, J. E. Holladay and H. Brown, Science 316 (15): 1597-1600 (2007). 